\title{
Prevalence of open- angle glaucoma in Iceland: Reykjavik Eye Study
}

F Jonasson', KF Damji', A Arnarsson',

T Sverrisson ${ }^{1}$, L Wang ${ }^{2}$, H Sasaki ${ }^{3}, K$ Sasaki ${ }^{3}$ and the Reykjavik Eye Study Group

\begin{abstract}
Purpose To establish the age- and sex-specific prevalence of open-angle glaucoma (OAG) subsuming pseudoexfoliation (PEX) in the city of Reykjavik.

Methods Participants 50 years of age and older who were part of the Reykjavik Eye Study and classified as having glaucoma were divided into three categories:

Category 1: two or more of the following based on optic nerve stereophotograph reading: vertical cup to disc ratio (VCDR) 97.5th percentile ( $>0.7$ ), focal glaucomatous disc change, C/D asymmetry of 97.5 th percentile difference between eyes $(>0.2)$ as well as glaucomatous visual field defect (GVFD). Category 2: 99.5th percentile of VCDR ( $>0.8)$ and $99.5 \%$ percentile difference between eyes ( $\geq 0.3$ ), without a GVFD.
\end{abstract}

Category 3: VA $<3 / 60$ and IOP $>99.5$ th percentile or $\mathrm{VA}<3 / 60$ and evidence of filtering surgery. For a glaucoma suspect, one of the following was present:

VCDR $>$ 99.5th percentile $(>0.8)$, focal glaucomatous disc change, $\mathrm{C} / \mathrm{D}$ asymmetry of 99.5th percentile ( $\geq 0.3)$, GVFD only, IOP $\geq 23 \mathrm{mmHg}$ (97.5 percentile). PEX was diagnosed by the presence of a central shield and/or a peripheral band on the anterior lens capsule.

Results Of 42 persons (22 males and 20 females) with OAG, $13(31.0 \%)$ had PEX. The minimum prevalence of OAG was $4.0 \%$ (42/1045) (95\% CI 2.8-5.2) for those 50 years and older and $10.3 \%$ (95\% CI 8.5-12.2) for PEX. The prevalence of OAG increases with age $(\mathrm{OR}=1.10 /$ year, $95 \%$ CI 1.07-1.13, $P=0.000)$ and the same applies for the prevalence of PEX, OR $=1.10$ (95\% CI 1.07-1.12, $P=0.000)$. Conclusion There is a $10 \%$ annual increase for both OAG and PEX in persons 50 years and older.

Eye (2003) 17, 747-753. doi:10.1038/

sj.eye. 6700374
Keywords: epidemiology; glaucoma;

pseudoexfoliation; end organ; damage

Introduction

The Icelandic population is almost exclusively Caucasian, predominantly descendants of settlers who arrived from Scandinavia and the British Isles about 1100 years ago. During the 20th century, several glaucoma surveys have been conducted in Iceland. ${ }^{1-5}$ Glaucoma was found to be the commonest cause of blindness, amounting to over $50 \%$ of all cases of blindness in $1950 .{ }^{5}$ Surveys in the 1980 s indicated that glaucoma might be the second most common cause of legal blindness in Iceland ${ }^{2-4}$ responsible for approximately $18 \%$ of blindness, and in 1998 this figure was down to 8\%. ${ }^{6}$

The purpose of the present study is to establish the age- and sex-specific prevalence of open-angle glaucoma (OAG), subsuming pseudoexfoliative glaucoma in the city of Reykjavik. This study is part of the Reykjavik Eye Study ${ }^{7-10}$ that addressed the same questions for age-related lens opacification and agerelated maculopathy.

\section{Materials and methods}

Following the guidelines of the Helsinki Declaration, appropriate ethical approvals were obtained from the Data Protection Commission and the Hospital Ethics Committee.

The participants were citizens of Reykjavik 50 years and older, and were randomly sampled using the National population census. A total of $6.4 \%$ people were sampled for each year of birth and both sexes. ${ }^{7-10}$ Of those 1700 thus sampled, $1379(81.1 \%)$ could be contacted and qualified of which 1045 (461 male and 584 females) elected to participate, giving a response rate of $75.8 \%{ }^{8}$ All were Caucasians. They were required to answer a questionnaire regarding lifestyle, health, previous disease, surgery, and medication. $^{7-10}$

\author{
${ }^{1}$ Department of \\ Ophthalmology \\ University Hospital \\ of Iceland \\ Landspítalinn, Iceland \\ ${ }^{2}$ University of Ottawa \\ Eye Institute \\ Ottawa, Canada \\ ${ }^{3}$ Kanasawa Medical \\ University \\ Uchinada, Japan \\ Correspondence: \\ F Jonasson \\ University Eye Department \\ Landspitalinn \\ 101 Reykjavik, Iceland \\ Tel: + 443545437217 \\ Fax: +44354560 2062 \\ E-mail: fridbert@ \\ landspitali.is
}




\section{Examination}

The site of the study was the University Eye Department in Reykjavik. All participants were examined utilizing a standard examination protocol including keratorefractometry (Nidek ARK 900), air puff tonometry (Nidek NT 2000, mean of three successful measurements), ${ }^{10}$ Scheimpflug photography of the anterior segment (Nidek EAS 1000), ${ }^{7}$ slit-lamp examination of the anterior segment, biomicroscopy of fundi with a 78D lens, and simultaneous stereo fundus photography centred on the optic disc and another on the fovea (Nidek 3Dx/NM). On the day of survey, gonioscopy was not done since this would have interfered with Scheimpflug and fundus photography. After maximal dilation of pupils, with Tropicamide 1\% and Phenylephrine $10 \%$ eye drops, lenses were examined for pseudoexfoliation (PEX) on the slit lamp by two experienced examiners. Only those eyes with a central shield and/or a peripheral band on the anterior lens capsule were considered to have definite PEX, and if they also had OAG they were considered to have pseudoexfoliative glaucoma.

A subgroup of 85 people $(8.1 \%)$ were recalled approximately 3 and 12 months later for full-threshold visual field assessment (octopus G1X) and gonioscopy (Goldmann single mirror lens at 3 months). This subgroup included all 45 people with a history of glaucoma (one each with angle closure and neovascular glaucoma and 43 with OAG) as well as 40 others considered to have the appearance of the optic disc suspect for glaucoma. The visual fields were evaluated in a masked fashion, by two glaucoma specialists (FJ/ThS). If the evaluation differed, the visual field was repeated and the two specialists were then required to reach a consensus. Grading of the optic discs was done by an experienced glaucoma specialist (KFD), in a masked fashion, using the simultaneous stereophotographs centred on the horizontal disc and vertical disc, and cup diameters were determined following the Beaver Dam Eye Study protocol. ${ }^{11,12}$ A plastic template with small circles ranging from $1 / 32(0.031)$ to $1 \frac{1}{4}(1.250)$ inches in diameter in $1 / 64(0.015)$ to $1 / 32(0.031)$ inch increments was used (Pickett, small circles no. 1203I) to grade disc and cup diameters from photographs. This was done by superimposing the circles over discs and cups measuring the largest cup to disc ratio within $20^{\circ}$ of the horizontal and vertical midline. The quality of photographs was assessed from 1 to 7 ( 1 and 2 represented categories that were too poor for assessment, and 3-7 represented varying degrees of improvement in quality). Of the 1045 people, 1026 had gradable stereophotographs for at least one eye, 1015 had gradable photographs for the right eye and 1014 for the left eye. Mean vertical cup to disc ratio
(VCDR) in our population is $0.4, \mathrm{SD} 0.23$, mode 0.5 , range 0.0-1.0.

\section{Definitions}

Definitions for OAG, category 1-3, and the glaucoma suspect are provided in Table 1 . Where visual fields were available, we utilized them in assigning diagnostic status. Those who were found not to have glaucoma or other conditions possibly affecting their optic nerve head were used in our survey to establish the 97.5th and 99.5th percentiles. Selection of VCDR $>0.7$ and $>0.8$ was based on the 97.5th and 99.5th percentiles, respectively. This is in keeping with suggestions made recently in the literature by Wolfs $e t a l^{13}$ and Foster $e t a l^{14}$ for the diagnosis of glaucoma in cross-sectional prevalence surveys. Also included are C/D asymmetry of the 97.5th percentile $(>0.2)$ and the 99.5 th percentile $(\geq 0.3)$ and focal glaucomatous disc changes. The lower numbers are criteria for category 1 and the higher numbers for category 2 (Table 1 ).

In healthy eyes the $97.5 \%$ percentile cutoff for intraocular pressure (IOP) was about $23 \mathrm{mmHg}$. Hence an IOP $\geq 23 \mathrm{mmHg}$ was considered abnormal and utilized as part of our definition of glaucoma suspect (GS). Eyes where IOP had been affected by medication, laser, or surgery were disregarded for this purpose.

The two eyes of a person are not independent regarding IOP and $C / D$, and for statistical purposes we therefore treated the right and left eyes separately in this article. When considering those with PEX, only those where the diagnosis was absolutely certain were accepted, namely where a central shield and/or a peripheral band were clearly seen. If there was any uncertainty, the eyes were listed as PEX suspects.

For statistical analysis we used multiple logistic regression, using the statistical package STATA (STATA Corp., 1997).

\section{Results}

Of the 1700 people in the random sample, 65 had died and 256 could not be located at their addresses indicated in the population census, apparently since they had moved without yet informing the Census Bureau. The remaining 1379 people could be located whereof 1045 elected to participate in all examinations and answer a questionnaire, giving a response rate of $75.8 \%$. Those unwilling to participate all answered a short questionnaire on the phone, among other things giving their reasons for non-participation. ${ }^{7}$

Table 2 shows the number, age and sex of the participants as well as age- and sex-specific minimum 
Table 1 Classification criteria used for open-angle glaucoma and glaucoma suspect

Diagnostic criteria for open-angle glaucoma (modified from Foster et al ${ }^{14}$ )

One of the following:

(1) Category 1 diagnosis (structural and functional evidence): 2/3 of the following criteria with a GVFD:

(a) Vertical cup to disc ratio $\geq 97.5$ th percentile $(>0.7)$

(b) Focal glaucomatous disc change (disc haemorrhage, notch of the neuroretinal rim, marked sloping of rim tissue, narrowest remaining rim of 0.1 disc diameter or less)

(c) $\mathrm{C} / \mathrm{D}$ asymmetry $\geq 97.5$ th percentile $(>0.2)$

(2) Category 2 diagnosis (structural evidence only with unproved field loss): $2 / 3$ of the following criteria:

(a) Vertical cup to disc ratio $\geq 99.5$ th percentile $(>0.8)$

(b) Focal glaucomatous disc change (disc haemorrhage, notch of the neuroretinal rim, marked sloping of rim tissue, narrowest remaining rim of 0.1 disc diameter or less)

(c) C/D asymmetry $\geq 99.5$ th percentile $(\geq 0.3)$

(3) Category 3 diagnosis (optic disc not seen, no field test): One of the following:

(a) $\mathrm{VA}<3 / 60$ and IOP $>99.5$ th percentile

(b) $\mathrm{VA}<3 / 60$ and the eye shows evidence of glaucoma filtering surgery

Diagnostic criteria for open-angle glaucoma suspect

One of the following:

(1) $\mathrm{IOP} \geq 23 \mathrm{mmHg}$

(2) IOP $\geq 23 \mathrm{mmHg}$ with $1 / 3$ of the following criteria for glaucomatous optic neuropathy (GON):

(a) Vertical cup to disc ratio $\geq 99.5$ th percentile

(b) Focal glaucomatous disc change (disc haemorrhage, notch of the neuroretinal rim, marked sloping of rim tissue, narrowest remaining rim of 0.1 disc diameter or less)

(c) $\mathrm{C} / \mathrm{D}$ asymmetry $\geq 99.5$ th percentile

(3) IOP $\geq 23 \mathrm{mmHg}$ with GVFD

(4) GVFD only

(5) $1 / 3$ criterion for GON listed in (2) above

Criteria for GVFD included ${ }^{14}$

(a) Asymmetric across the horizontal midline (in early/moderate cases)

(b) Located in the mid-periphery (in early/moderate cases)

(c) Clustered in neighbouring test points

(d) Not explained by any other disease

(e) Considered a valid representation of the subject's functional status (based on performance indices such as false positive rate)

Notes: In diagnosing category 1 or 2 glaucoma, alternative explanations for CDR findings or VF findings were excluded.

Exclusion criteria for OAG and for OAG suspects included other causes for glaucoma (eg uveitis, angle recession), corticosteroid use greater than 1 month (topical or systemic), iris abnormality other than peripupillary atrophy, past history of blunt or sharp trauma.

Table 2 Age- and sex-specific prevalence (\%) of OAG and 95\% confidence intervals

\begin{tabular}{|c|c|c|c|c|c|c|c|c|c|}
\hline \multirow[b]{2}{*}{ Age (years) } & \multicolumn{3}{|c|}{ People examined } & \multicolumn{6}{|c|}{ People with OAG } \\
\hline & Males & Females & $M+F$ & $M$ & $M \%(95 \% C I)$ & $F$ & $F \%(95 \% C I)$ & $M+F$ & $M+F \%(95 \% C I)$ \\
\hline $50-59$ & 167 & 195 & 362 & 2 & $1.2(0.0-2.9)$ & 0 & 0 & 2 & $0.6(0.0-1.3)$ \\
\hline $60-69$ & 144 & 210 & 354 & 4 & $2.8(0.1-5.5)$ & 6 & $2.9(0.6-5.1)$ & 10 & $2.8(1.1-4.6)$ \\
\hline $70-79$ & 116 & 135 & 251 & 10 & $8.6(3.4-13.8)$ & 10 & $7.4(2.9-11.9)$ & 20 & $8.0(4.6-11.3)$ \\
\hline \multirow[t]{2}{*}{$80+$} & 34 & 44 & 78 & 6 & $17.6(4.1-31.1)$ & 4 & $9.1(0.2-17.9)$ & 10 & $12.8(5.5-21.2)$ \\
\hline & 461 & 584 & 1045 & 22 & $4.8(2.8-6.7)$ & 20 & $3.4(1.9-4.9)$ & 42 & $4.0(2.8-5.2)$ \\
\hline
\end{tabular}

per cent prevalence and confidence intervals of glaucoma according to the definition outlined in Table 1.

The distribution of VCDR can be seen in Figure 1.

Besides normal variation, causes for optic neuropathy other than glaucoma found were past history of temporal arteritis, cerebrovascular accident, macular degeneration, and Lebers optic atrophy. These last were excluded when establishing disc criteria for glaucoma diagnosis. 


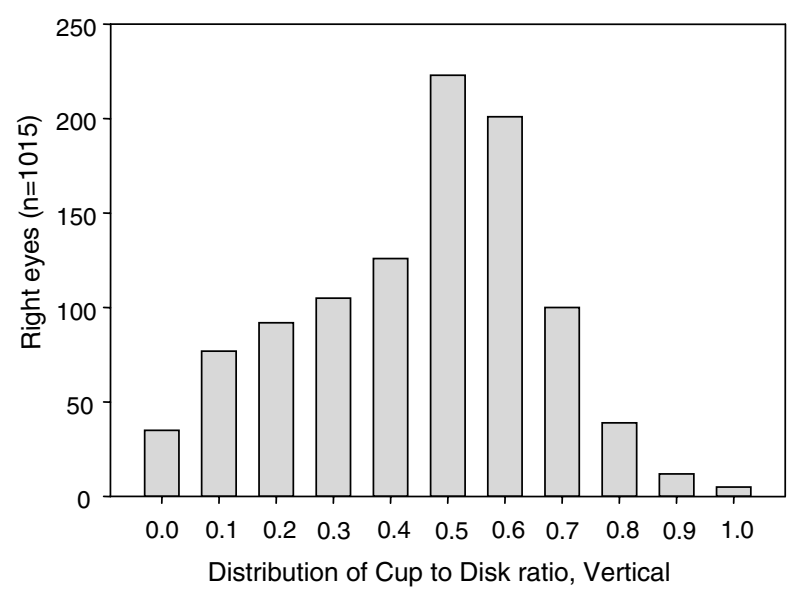

Figure 1 Distribution of vertical cup to disc ratio, right eyes.

We analysed IOP for healthy right eyes to establish $97.5 \%$ cutoff which was about $23 \mathrm{mmHg}$, and IOP $\geq 23 \mathrm{mmHg}$ was used as a part of our definition of glaucoma suspects. The results for the left eyes were similar. ${ }^{10}$ The diagnosis of OAG in 42 people (Table 2) was based on category 1 criteria in 17 people, including $2 / 3$ disc criteria and glaucomatous visual field defect. Seven $(41.2 \%)$ of the last also had PEX. Twenty-five were diagnosed on criteria for category 2 , ie without visual fields (19) or without visual field defect (6). No participant was classified to belong to category 3 , since none had VA $<3 / 60$ related to OAG. Age- and sexspecific prevalences for the 123 people considered to be GSs according to the criteria outlined are shown in Table 3. Two had IOP $\geq 23 \mathrm{mmHg}$ and $1 / 3$ disc criteria, 13 had IOP $\geq 23 \mathrm{mmHg}$ only and 95 had disc criteria only.

One participant who had IOPs of $25 \mathrm{mmHg}$ in the right eye and $23 \mathrm{mmHg}$ in the left eye was considered to have glaucomatous optic neuropathy and was, on visual field testing, found to have bitemporal visual field defect and, on computerized tomography, to have pituitary tumour that was removed surgically, uneventfully, within 1 month of the survey.

Forty-three people had a history of OAG and had received pressure-lowering treatment, some possibly for ocular hypertension. Seventeen of the 43 with a history of glaucoma were identified among the 42 persons diagnosed with glaucoma all in category 1 criteria, a further 16 were considered GSs, and 10 participants remained unidentified by our criteria and may have been treated for ocular hypertension. Of the 42 people diagnosed with glaucoma in our survey, 13 were considered to have normal tension glaucoma (31.0\%) having IOP $<21 \mathrm{mmHg}$ without pressure-lowering treatment.

On review of stereo optic nerve photographs, 10 females and two males had optic disc haemorrhage in one eye, and were classified as OAG suspects. None of these patients had other disc or visual field features to suggest definitive OAG classification.

Definite PEX, ie characteristic fibrillar pseudoexfoliative material visualized on the lens capsule on slit-lamp examination after dilatation by both examiners, was found in 145 eyes, 108 people, 93 female and 52 male eyes. Of those 42 diagnosed by our definition as having glaucoma, $13(31.0 \%)$ had definite PEX and a further seven (16.7\%) were suspected of having PEX. Thus, altogether 20 (47.6\%) of the 42 people with definite diagnosis of OAG had either definite PEX or were suspected of having PEX. Regarding 123 GSs, definite PEX was found in 23 people (18.4\%), and in the group (861) having neither glaucoma nor being GSs $8.4 \%$ had PEX. Definite PEX in either eye greatly increased with age from $2.5 \%$ of all people in the 50 s to $33.3 \%$ in the 80s (Table 4). Multiple regression analysis shows that 1 year ageing increases the risk of developing PEX by $10 \%$ (OR 1.10, 95\% CI 1.07-1.12, $P=0.000$ ). After correcting for age, the OR for women as compared with men is 1.5\% (95\% CI 1.0-2.3), $P<0.06$. Multiple regression analysis also indicates a $10 \%$ increase per year for OAG (OR 1.10, 95\% CI 1.07-1.13, $P=0.000$ ). After correcting for age, PEX is found to be a risk factor for being classified as a GS, OR 1.95 (CI 1.14-3.35), $P=0.015$, while it is not a significant risk factor for glaucoma according to our definition.

\section{Discussion}

We utilized data from the Reykjavik Eye Study to examine the prevalence of OAG and PEX in Reykjavik, Iceland. The response rate is considered acceptable

Table 3 Age- and sex-specific prevalence (\%) of GSs (95\% confidence intervals)

\begin{tabular}{lrrrrrr}
\hline Age (years) & $M$ & $\%(95 \% C I)$ & $F$ & $\%(95 \% C I)$ & $M+F$ & $\%(95 \% C I)$ \\
\hline $50-59$ & 16 & $9.6(5.1-14.1)$ & 24 & $12.3(7.7-17.0)$ & 40 & $11.0(7.8-14.3)$ \\
$60-69$ & 15 & $10.4(5.4-15.5)$ & 19 & $9.0(5.1-13.0)$ & 34 & $9.6(6.5-12.7)$ \\
$70-79$ & 8 & $6.9(2.2-11.6)$ & 25 & $18.5(11.9-25.2)$ & 33 & $13.1(8.9-17.4)$ \\
$80+$ & 6 & $17.6(4.1-31.1)$ & 10 & $22.7(9.8-35.6)$ & 16 & $20.5(11.3-29.7)$ \\
& 45 & $9.8(7.0-12.5)$ & 78 & $13.4(10.6-16.1)$ & 123 & $11.8(9.8-13.7)$ \\
\hline
\end{tabular}


Table 4 Age- and sex-specific prevalence (\%) of pseudoexfoliation and 95\% confidence intervals

\begin{tabular}{lcccccc}
\hline Age (years) & $M$ & $\%(95 \% C I)$ & $F$ & $\%(95 \% C I)$ & $M+F$ & $\%(95 \% C I)$ \\
\hline $50-59$ & 2 & $1.2(0.0-2.9)$ & 7 & $3.6(1.0-6.2)$ & 9 & $2.5(0.9-4.1)$ \\
$60-69$ & 12 & $8.3(3.8-12.9)$ & 19 & $9(5.1-13.0)$ & 31 & $8.8(5.8-11.7)$ \\
$70-79$ & 15 & $12.9(6.7-19.1)$ & 27 & $20(13.2-26.8)$ & 42 & $16.7(12.1-21.4)$ \\
$80+$ & 10 & $29.4(13.3-45.5)$ & 16 & $36.3(21.6-51.2)$ & 26 & $33.3(22.6-44.0)$ \\
& 39 & $8.5(5.9-11.0)$ & 69 & $11.8(9.2-14.4)$ & 108 & $10.3(8.5-12.2)$ \\
\hline
\end{tabular}

(75.8\%). Our study included institutionalized patients in the oldest age group, which differs from some studies that exclude this group. ${ }^{15}$ It was not possible to follow the examination protocol outside the hospital, ie to do visual field testing, fundus photography, and Scheimpflug photography. The consequence is a considerable dropout among those 80 years and older as a result of decreased mobility.

For the definition of glaucoma, we decided to utilize a structural criterion based on objective reading of stereophotographs of the optic disc and, when available, incorporate a functional definition to strengthen the diagnostic classification. This approach and definition is in keeping with recent studies, ${ }^{13,14}$ and suggestions of an expert working group, for the diagnosis of glaucoma in cross-sectional prevalence surveys. ${ }^{14}$ The fundamental concept is that the term glaucoma is reserved for a significant end organ damage. Our results for the 97.5th (0.7) and 99.5th percentiles (0.8) are remarkably similar to the results of previous studies. ${ }^{13,14}$ IOP is not a part of this definition, although it is included as a criterion for GSs. Of 43 people who had received treatment for glaucoma prior to the survey, 17 were identified in the present survey as having glaucoma, all in category 1 . A further 16 people were diagnosed as GSs and 10 remained unidentified and should probably be classified as ocular hypertensives.

Normal tension glaucoma previously considered uncommon in Iceland ${ }^{1-4}$ was found in 13 participants $(1.2 \%)$ and it constitutes $31.0 \%$ of OAG in this study. Two of those had been on systemic and topical corticosteroid treatment for some time in the past, which may have led to temporary pressure rise and consequent glaucomatous optic neuropathy. A third participant had cerebral basal ganglia degeneration, motor and non-motor, and the fourth had Bernard-Souliere syndrome, both conditions possibly affecting the appearance of the optic disc and visual fields. For the remainder, arterial hypertension and treatment thereof were commonly found.

Comparing the present prevalence data with previous Icelandic data, ${ }^{3}$ we find the prevalence of OAG similar for those 50 to 69-year-olds, while the figures for those 70 years and older are somewhat lower in the present study. This may be associated with different criteria since the previous study used the Framingham Eye Study criteria. ${ }^{16}$ Altogether 42 people are considered to have OAG in our survey, a minimum per cent prevalence of $4.0 \%$ for those 50 years and older. In Nottingham in England, $1.3 \%$ of the population 50 years and older were found to be on glaucoma medication and a further $1.4 \%$ were diagnosed as having OAG and put on treatment. ${ }^{17}$ Another English survey ${ }^{18}$ found prevalence in the age group 76-84 years to be $7.1 \%$, compared with 9.8\% (95\% CI 4.9-14.7) in this study. Neither of the English studies mentions PEX. In the Baltimore Eye Study the prevalence of glaucoma is $1.3 \%$ for the white population, ${ }^{19}$ and in Roscommon in Ireland $2 \%{ }^{20}$ for those 50 years and older. Since diagnostic criteria differ somewhat between studies, direct comparison may be fallacious.

We did not obtain a visual field on all participants and this is a shortcoming of our study. However, in epidemiological studies, visual fields may give relatively high false positive and false negative results, particularly in the older population. ${ }^{12,21}$ Thus in the Rotterdam Eye Study, $18.4 \%$ of the participants failed the initial visual field screening whereby $1.1 \%$ were eventually deemed to have glaucoma on visual field criteria. ${ }^{21}$ Foster and coworkers, considering visual field testing in prevalence surveys, suggested that around $33 \%$ false negative rate was not uncommonly found, with $95 \%$ CI 13 to $53 \% .^{14}$

We used a Nidek NT2000 non-contact airpuff tonometer to measure IOP. This instrument has been shown to give similar results to the Goldmann applanation tonometer ${ }^{22}$ with slightly more variability. We used the mean of three successful measurements, outlying measurements being disregarded. Non-contact tonometry has the benefit of avoiding anaesthesia and the risk of infection and abrasion and does not interfere with subsequent examination of the survey. Our IOP results in healthy eyes are similar to those of many previous studies, ${ }^{1,12,23-26}$ using Schiötz and applanation tonometry.

There are arguments for and against including people with glaucoma and PEX syndrome and pigment dispersion syndrome as cases of OAG. We have included them as a variant of OAG rather than classifying them as 
secondary glaucomas, though this view remains to be fully vindicated. ${ }^{14}$

We have shown in a previous article that PEX is a common and familial condition in Iceland, probably genetically inherited..$^{27}$ The prevalence of OAG subsuming pseudoexfoliative glaucoma is $5.4 \%$ for 65 to 74 -year-olds in the present study, $5.3 \%$ in the mid-Norway Eye Study, ${ }^{28}$ and $5.7 \%$ in a Swedish study, ${ }^{29}$ all populations with a high prevalence of PEX. Like in all previous studies from Iceland, narrow-angle glaucoma is rare, ${ }^{1-4}$ only one case being identified.

Interestingly, we did not come across any cases of pigment dispersion syndrome. For a definite diagnosis of OAG in early stages, several visits to an ophthalmologist over time may be necessary. In a prevalence survey it may, however, be possible to identify these persons as GSs for further follow-up. It is of interest in the present survey that contrary to glaucoma and PEX, there is no significant age-related increase in the prevalence of GSs. It is also of interest that PEX, while being a significant risk factor for GSs is not a significant risk factor for OAG using the present definition. Analysing, however, those 43 people with a history of OAG and pressure-lowering treatment, PEX is found to be an important risk factor for OAG, OR 4.1 (95\% CI 2.1-8.1, $P<0.001)$. It is not clear if this discrepancy is the result of ocular hypertensives being classified as OAG in the group of those with history of OAG or if the present definition is less suited for populations with a high prevalence of PEX or both.

\section{Acknowledgement}

We are indebted to Laufey Tryggvadottir (MSc) for assistance regarding epidemiology and statistics. We are grateful to Nidek Co. Ltd (Japan) for providing equipment and technical staff for the survey. This project was supported by grants from St Joseph's Hospital, Landakot Foundation, Reykjavik, Iceland, University National Hospital, University of Iceland Research Grant and The Icelandic Research Council.

\section{References}

1 Björnsson G. Primary glaucoma in Iceland. Acta Ophthalmol (Copenh) 1967; (Suppl 91): 68.

2 Viggosson G, Björnsson G, Ingvarsson JG. The prevalence of open-angle glaucoma in Iceland. Acta Ophthalmol (Copenh) 1986; 64: 138-141.

3 Jonasson F, Thordarson K. Prevalence of ocular disease and blindness in a rural area in the eastern region of Iceland during 1980 through 1984. Acta Ophthalmol 1987; 65 (Suppl 182): $40-43$

4 Sverrisson T, Viggosson G, Björnsson G. Visual impairment in patients with chronic open angle glaucoma. Acta Ophthalmol 1990; 68 (Suppl 195): 71-73.
5 Björnsson G. Prevalence and causes of blindness in Iceland. Am J Ophthalmol 1955; 39: 202-208.

6 The Low Vision Institute in Iceland. Annual Report 1998. Blind Registration, Reykjavik, 1999, p 24.

7 Sasaki H, Jonasson F, Kojima M, Katoh N, Ono M, Sasaki K, The Reykjavik Eye Study Group. Reykjavik Eye Study. Prevalence of lens opacification with reference to identical Japanese studies. Ophthalmologica 2000; 214: 412-420.

8 Katoh N, Jonasson F, Sasaki H, Kojima M, Ono M, Sasaki K, The Reykjavik Eye Study Group. Cortical lens opacification in Iceland Risk factor analysis-Reykjavik Eye Study. Acta Ophthalmol Scand 2000; 78: 642-646.

9 Arnarsson A, Jonasson F, Sasaki H, Ono M, Jonsson V, Kojima $\mathrm{M}$ et al. Risk factors for nuclear lens opacification. The Reykjavik Eye Study. In: Takahashi N, Kojima M, Sliney $\mathrm{DH}$, Hockwin O (eds). Progress in Lens and Cataract Research. Dev Ophthalmol 2002; 35: 12-20.

10 Eysteinsson Th, Jonasson F, Sasaki H, Arnarsson A, Sverrisson Th, Sasaki K et al. Central corneal thickness, radius of the corneal curvature and intraocular pressure in normal subjects using non-contact techniques: Reykjavik Eye Study. Acta Ophthalmol Scand 2002; 80: 11-15.

11 Klein BEK, Moss SE, Magli YL, Klein R, Johnson JC, Roth H. Optic disc cupping as clinically estimated from photographs. Ophthalmology 1987; 94: 1481-1483.

12 Klein BEK, Klein R, Sponsel WE, Franke T, Cantor LB, Martone J et al. Prevalence of glaucoma. The Beaver Dam Eye Study. Ophthalmology 1992; 99: 1499-1504.

13 Wolfs RCW, Borger PH, Ramrattan RS, Klaver CCW, Hulsman CAA, Hofman A et al. Changing views on open-angle glaucoma: definitions and prevalences-The Rotterdam Study. Invest Ophthalmol Vis Sci 2000; 41: 3309-3321.

14 Foster PJ, Buhrmann R, Quigley HA, Johnson GJ. The definition and classification of glaucoma in prevalence surveys. Br J Ophthalmol 2002; 86: 238-242.

15 Mitchell P, Smith W, Attebo K, Healey PR. Prevalence of open angle glaucoma in Australia. The Blue Mountain Eye Study. Ophthalmology 1996; 103: 1661-1669.

16 Leibowitz HM, Krueger DE, Maunder LR, Milton RC, Kini MM, Kahn HA et al. The Framingham Eye Study monograph: an ophthalmological and epidemiological study of cataract, glaucoma, diabetic retinopathy, macular degeneration, and visual acuity in a general population of 2631 adults, 1973-1975. Surv Ophthalmol 1980; 24 (Suppl): 335-610.

17 Vernon SA, Henry DJ, Cater L, Jones SJ. Screening for glaucoma in the community by non-ophthalmologically trained staff using semi automated equipment. Eye 1990; 4: 89-97.

18 Gibson JM, Rosenthal AR, Lavery J. A study of the prevalence of eye disease in the elderly in an English community. Trans Ophthalmol Soc UK 1985; 104: 196-203.

19 Tielsh J, Sommer A, Katz J, Royall X, Quigley H, Javitt J (Baltimore Eye Study). Racial variation in the prevalence of primary open angle glaucoma. JAMA 1991; 226: 369-374.

20 Coffey M, Reidy A, Wormald R, Xian WX, Wright L, Courtney P. Prevalence of glaucoma in the west of Ireland. Br J Ophthalmol 1993; 77: 17-21.

21 Dielemans I, Vingerling JR, Wolfs RCW, Hofman A, Grobbee DE, de Jong PTVM. The prevalence of primary open-angle glaucoma in a population based study in the 
Netherlands. The Rotterdam Study. Ophthalmology 1994; 101: 1851-1855.

22 Cho P, Lui T. Comparision of the performance of Nidek NT2000 non-contact tonometer with the Keeler pulsair 2000 and the Goldmann applanation tonometer. Optometry Vis Sci 1997; 74: 51-58.

23 Leske MC, Connell AMS, Wu Sy, Hyman L, Schachat AP. Distributions of intraocular pressure. Barbados Eye Study. Arch Opthalmol 1997; 115: 1051-1057.

24 Hirvelea H, Tuulonen A, Laatikainen L. Intraocular pressure and prevalence of glaucoma in elderly people in Finland: a population based study. Int Ophthalmol 1995; 18: 299-307.

25 Hollows FC, Graham PA. Intra-ocular pressure, glaucoma, and glaucoma suspects in a defined population. $\mathrm{Br} \mathrm{J}$ Opthalmol 1966; 50: 570-586.
26 Leydhecker W, Akiyama K, Neumann HG. Der intraokulere Druck gesunder menschlicher Augen. Klin Monatsbl Augenheilkd 1958; 133: 662-670.

27 Allingham R, Loftsdottir M, Gottfredsdottir MS, Thorgeirsson E, Jonasson F, Sverrisson Th et al. Pseudoexfoliation syndrome in Icelandic families. Br J Ophthalmol 2001; 85: 702-707.

28 Ringvold A, Blika S, Elsås T, Guldahl J, Brevik T, Hesstvedt $\mathrm{P}$ et al. The middle-Norway eye-screening study II.

Prevalence of simple \& capsular glaucoma. Acta Ophthalmol 1991; 69: 273-280.

29 Bengtsson B. The prevalence of glaucoma. $\mathrm{Br}$ J Ophthalmol 1981; 65: 46-49. 